\title{
Fundamental parameters of Be stars located in the seismology fields of COROT ${ }^{\star}, \star \star$
}

\author{
Y. Frémat ${ }^{1}$, C. Neiner ${ }^{2,3}$, A.-M. Hubert ${ }^{3}$, M. Floquet ${ }^{3}$, J. Zorec ${ }^{4}$, \\ E. Janot-Pacheco ${ }^{5}$, and J. Renan de Medeiros ${ }^{6}$
}

\author{
1 Royal Observatory of Belgium, 3 avenue circulaire, 1180 Brussels, Belgium \\ e-mail: yves.fremat@oma.be \\ 2 Institute for astronomy, KU Leuven, Celestijnenlaan 200B, 3001 Leuven, Belgium \\ 3 GEPI/UMR 8111 du CNRS, Observatoire de Paris-Meudon, 5 place Jules Janssen, 92195 Meudon, France \\ 4 Institut d'Astrophysique de Paris, UMR 7095 CNRS, Université Pierre \& Marie Curie, 75014 Paris, France \\ 5 Instituto de Astronomia, Geofísica e Ciências Atmosféricas, Universidade de São Paulo, Rua do Matão 1226, 05508-090 São Paulo, \\ Brazil \\ 6 Departamento de Fisica, Universidade Federal do Rio Grande do Norte, 59072-970 Natal, Brazil
}

Received 25 April 2005 / Accepted 21 October 2005

\begin{abstract}
In preparation for the COROT space mission, we determined the fundamental parameters (spectral type, temperature, gravity, $V$ sin $i$ ) of the Be stars observable by COROT in its seismology fields (64 Be stars). We applied a careful and detailed modeling of the stellar spectra, taking into account the veiling caused by the envelope, as well as the gravitational darkening and stellar flattening due to rapid rotation. Evolutionary tracks for fast rotators were used to derive stellar masses and ages. The derived parameters will be used to select Be stars as secondary targets (i.e. observed for 5 consecutive months) and short-run targets of the COROT mission. Furthermore, we note that the main part of our stellar sample falls in the second half of the main sequence life time, and that in most cases the luminosity class of Be stars is inaccurate in characterizing their evolutionary status.
\end{abstract}

Key words. stars: emission-line, $\mathrm{Be}$ - stars: activity - stars: fundamental parameters - stars: statistics

\section{Introduction}

\subsection{Be stars}

Be stars are non-supergiant B stars that show or have shown $\mathrm{H} \alpha$ emission (Jaschek et al. 1981). Emission does not only occur in the first members of the Balmer line series, but can affect the continuum and line profiles of other atoms or ions, such as Fe II. It is generally agreed that, in classical Be stars, this emission is due to the presence of a cool, disk-like circumstellar envelope concentrated in the equatorial plane. For a complete review of the "Be phenomenon" and its characteristics, see Porter \& Rivinius (2003).

Classical Be stars are fast rotators. Statistical studies suggest that their angular speed ranges from 60 to $100 \%$ of the critical breakup velocity $\left(\Omega_{\mathrm{c}}\right)$, with a narrow maximum occurrence at $80 \%$ (Chauville et al. 2001) or at $90 \%$ when accounting for fast rotation effects (Frémat et al. 2005) in order to limit the

* Based on GAUDI, the data archive and access system of the ground-based asteroseismology programme of the COROT mission. The GAUDI system is maintained at LAEFF (http://ines.laeff.esa.es/corot/). LAEFF is part of the Space Science Division of INTA. Also based on OHP (Observatoire de Haute-Provence, France), LNA (Laboratório Nacional de Astrofísica, Brazil) and FEROS (ESO, 072.D-0315(A)) observations.

$\star \star$ A part of the tables (Full Tables 1, 4, 5 and 7) and figures described in this paper are available at the CDS via anonymous ftp to cdsarc.u-strasbg.fr (130.79.128.5) or via http://cdsweb.u-strasbg.fr/cgi-bin/qcat?J/A+A/451/1053 saturation of the lines' FWHM at high rotation rates (Townsend et al. 2004).

Although the envelopes of classical Be stars are probably formed during episodes of strong mass ejection, the precise origin of these ejections is still unknown and may differ from star to star. A currently interesting explanation is fast (non-critical) rotation and the beating of non-radial pulsation (NRP) modes combining their effects to cause matter expulsion. In the H-R diagram, early Be stars are indeed located at the lower border of the instability strip of the $\beta$ Cep stars, while mid and late Be stars are mixed with Slowly Pulsating B (SPB) stars. Short and long period pulsations are therefore expected and confirmed in the majority of Be stars, except the late sub-classes. From the Hipparcos database (Hubert \& Floquet 1998) it was shown that short-term variability is present in almost all early-Be stars $(86 \%)$, while it seems to be less common in the cooler ones $(40 \%$ to $18 \%$ from the mid to the latest spectral types). This fact, however, could be due to the level of variability detection of current instrumentation, since the amplitude of pulsations in late subtypes of B stars is expected to be very small (a few mmag). Up to now, about hundred Be stars have been claimed as shortterm periodic variables (hours, tens of hours) and their number is increasing. However, the detection of short and long-term pulsations is difficult using ground-based observations even in the framework of multisite campaigns (e.g. the Musicos 98 campaign, see Neiner et al. 2002). The prediction and the study of the coincidence between the beating of multiperiodic pulsations and the occurrence of mass-ejection further needs the determination of accurate periods and the detection of the most complete 
sample of pulsation modes, which is not easy, even during multisite campaigns. Although multiperiodicity has been detected in several Be stars (e.g. 66 Oph, Floquet et al. 2002), only one such coincidence between beating and matter ejection has been reported ( $\mu$ Cen, Rivinius et al. 1998). Most of the presently known cases of pulsation in Be stars result from high-resolution spectroscopy. This technique is very powerful in identifying highorder pulsation modes, but not well adapted to resolve closely spaced multiple periods. Photometry with COROT will therefore provide a much more superior observing tool to detect new multiperiodic pulsators among Be stars.

The COROT mission will provide 5 months of continuous observations of a substantial number of Be stars. It will allow us to reach an unparalleled precision in the determination of pulsation frequencies, giving us the opportunity to study their possible magnetic splitting and the rotational modulation in Be stars.

\subsection{The COROT mission}

The COROT (COnvection, ROtation and planetary Transits) satellite ${ }^{1}$ will be launched in August 2006 and has two goals: to study the interior of stars by looking at their oscillations and to search for extrasolar planets by detecting planetary transits (see Baglin et al. 2002). Four CCDs are used: two for the asteroseismology program and two for the exoplanetary program. The asteroseismology CCDs are positioned on the sky to simultaneously observe one (or sometimes two) bright $(V \sim 5.5)$ primary target(s) plus eight or nine surrounding secondary targets with a magnitude $5.6 \leq V \leq 9.4$.

The $30 \mathrm{~cm}$ telescope of COROT will be pointed alternatively towards the galactic centre and anticentre. These two cones of observations, with a radius of 10 degrees, are the pointing limits for the CCDs. The two asteroseismology CCDs cover a field of view of $1.3 \times 2.6$ degrees. At least 5 such fields will be observed by COROT for $\sim 5$ months each. Also, short observing run (2030 days) will be performed on specific targets so that the full target list covers as well as possible the HR diagram.

Bright Be stars can therefore be observed either as secondary targets of the asteroseismology program in long runs, or as short runs targets. An international collaboration led by A.-M. Hubert is preparing these observations. In this paper, we present the procedure that was carried out to perform a preliminary analysis of as many bright $(5.6<V<9.4)$ classical Be stars as possible in the observing cones of COROT. The position, spectral type and variability of these Be stars are factors taken into account for the selection of the fields that will be observed by COROT. To carry out this first target selection, we therefore need good stellar parameters with typical accuracies of $10 \%$ on the effective temperature $\left(T_{\text {eff }}\right)$, of 0.1 to 0.2 dex on the surface gravity $(\log g)$, and of 5 to $10 \%$ on the projected rotation velocity $(V \sin i)$. To be valuable, the parameter determination further needs to account for the peculiar nature of Be stars, including the effects of fast rotation, circumstellar emission and departure from LTE.

Most of the data used in this study are available in the GAUDI (Ground-based Asteroseismology Uniform Database Interface) database (Solano et al. 2005) and are described in Sect. 2. In GAUDI, more specific information, such as fundamental stellar parameters (surface gravity and effective temperature) and projected rotation velocities, can also be found and were automatically derived from the observations. However, these parameters were obtained without accounting for either circumstellar emission or fast rotation effects, which are

\footnotetext{
${ }^{1}$ http://corot .oamp.fr/
}

generally expected to affect the spectra of Be stars. The goal of the present work is therefore to re-estimate the effective temperature $\left(T_{\text {eff }}\right)$, surface gravity (log $g$ ) and projected rotation velocity $(V \sin i)$ accounting, as far as possible, for the peculiar nature of Be stars, in order to update the GAUDI database and thus facilitate the target selection for COROT.

For the same reasons, this work also aims to identify the stars in our sample that could be considered as particular, such as Herbig stars or spectroscopic binaries with a Be star component. The model atmospheres we used are defined in Sect. 3, while the procedure we adopted to perform these determinations is detailed in Sect. 4. Our results are given in Sect. 5, with remarks concerning several specific targets in Sect. 6, and discussed in Sect. 7 .

\section{Observations}

In preparation for the COROT satellite observations, an ambitious ground-based observing program was performed (P.I.: C. Catala, Observatoire de Paris). For each star with a magnitude between 5.6 and 8 located in the observing cones of the COROT satellite, at least one spectrum was obtained and stored in the GAUDI database. The sample of stars we are studying in the present paper is therefore a sub-sample of the compilation by Solano et al. (2005). It gathers the targets that are well-known Be stars or that were recently identified as Be stars (Neiner et al. 2005).

The data were mainly obtained with two high-resolution échelle spectrographs $(R \sim 40000-50000)$ : ELODIE at the $2 \mathrm{~m}$ telescope of the Observatoire de Haute-Provence (OHP, France) and FEROS at the $1.5 \mathrm{~m}$ and $2.2 \mathrm{~m}$ telescopes of ESO (La Silla, Chile). Additional observations were also obtained at the $1.9 \mathrm{~m}$ telescope at SAAO (South Africa) with the GIRAFFE spectrograph, with the CORALIE spectrograph on the $1.2 \mathrm{~m}$ Swiss telescope in La Silla (Chile), with the SARG (Spectrografo Alta Risoluzione Galileo) spectrograph at the $3.6 \mathrm{~m}$ Telescopio Nazionale Galileo (TNG, La Palma, Spain) and with the Coudé spectrograph on the $2 \mathrm{~m}$ telescope of the Tautenburg observatory (Germany).

To complete the sample of Be stars in the observing cones of COROT, additional spectra of Be stars with a magnitude between 8 and 9.4 were obtained: in Brazil, at the LNA (Laboratório Nacional de Astrofisica, Observatório do Pico dos Dias) with the Cassegrain spectrograph (OPD CASS) attached to the $1.6 \mathrm{~m}$ Boller \& Chivens telescope and using the 900 lines/mm grating $(R \sim 7000)$; in France, at the OHP (Observatoire de Haute-Provence) with AURELIE (1.52-m telescope) at medium resolution $(R \sim 15000$ with the grating No. 2 , $\Delta \lambda=220 \AA)$ and lower resolution $(R \sim 7000$ with the grating No. 3, $\Delta \lambda=440 \AA$ ); and in Chile with FEROS (ESO) on Brazilian observing time.

In principle, the sample of studied stars contains all Be stars in the observing cones of COROT with adequate magnitude $(5.6 \leq V \leq 9.4)$. The target list was compiled using SIMBAD and adding newly discovered Be stars from Neiner et al. (2005). However, known SB2 with a Be star component and interacting Be binaries were rejected from the list of possible Be targets. A few faint Be stars are also not studied in this paper because no spectra were obtained due to bad weather conditions. As a consequence, the sample of studied Be stars is not complete, but no systematic bias is expected. In particular, the samples in the centre and anticenter direction are equivalent. 


\section{Model atmospheres and flux grids}

The plane-parallel atmosphere models we used for effective temperatures ranging from $15000 \mathrm{~K}$ to $27000 \mathrm{~K}$ were computed in two consecutive steps. To account in the most effective way for line-blanketing, the temperature structure of the atmospheres was computed by Kurucz (1993) using the ATLAS9 FORTRAN program. Non-LTE level populations were then calculated for each of the atoms we considered using TLUSTY (Hubeny \& Lanz 1995) and keeping the temperature and density distributions fixed. The surface chemical abundances we adopted are those published by Grevesse \& Sauval (1998) for the Sun.

Table 2 lists the ions we introduced in the computations. Except for C II, the atomic models we used in this work were downloaded from TLUSTY's homepage ${ }^{2}$ maintained by Hubeny and Lanz. C II was treated with the MODION IDL package developed by Varosi et al. (1995) and by adopting the atomic data (oscillator strengths, energy levels and photoionization cross sections) selected from the TOPBASE database (Cunto et al. 1993). It reproduces the results obtained by Sigut (1996).

Model atmospheres with effective temperatures lower than $15000 \mathrm{~K}$ were treated assuming full LTE, while those hotter than $27000 \mathrm{~K}$ were taken from the OSTAR2002 NLTE grid (Lanz \& Hubeny 2003). The grid of fluxes we use during the fitting procedure (Sect. 4.1) was built with SYNSPEC and for effective temperatures and surface gravities ranging from 8000 to $50000 \mathrm{~K}$ and from 2.5 to $4.5 \mathrm{dex}$ (cgs) respectively.

\section{Adopted procedure}

To take into account the main phenomena expected to affect the spectra of Be stars, the determination of their fundamental parameters was carried out in three consecutive steps, each of them being described in the following sections and summarized in Fig. 2.

\subsection{Apparent fundamental parameters determination}

Hydrogen, helium and $\mathrm{Mg}$ II lines are generally assumed to be good temperature and gravity indicators for the study of B-type stars. Figure 1 shows the variation of the equivalent width computed for the He I 4471 and Mg II 4481 spectral lines with effective temperature and surface gravity. Their different broadening mechanisms and transition probabilities further present the advantage of having been studied in detail for a long time, allowing accurate line profile computations. In our procedure, we therefore mainly focus on a spectral domain ranging from 4000 to $4500 \AA$ (see line-identification and computed equivalent widths in Table 3 ), which gathers no less than two hydrogen lines, 5 strong helium lines and 2 blended $\mathrm{Mg}$ II lines. Observations obtained in this region, and for each of the considered Be stars, are compared to a grid of synthetic spectra (see Steps 1 and 2 of Fig. 2). For efficiency reasons, this comparison is performed with a least squares method based on the MINUIT minimization package developed at CERN, which we transposed to a FORTRAN computer code named GIRFIT that treats large datasets. GIRFIT interpolates the spectra in a grid of stellar fluxes computed with plane-parallel model atmospheres (see Sect. 3) for different values of the effective temperature and of the surface gravity. To account for the instrumental resolution and the Doppler broadening due to rotation, the spectra are then convolved with a Gaussian function using subroutines

${ }^{2}$ http://tlusty.gsfc.nasa.gov

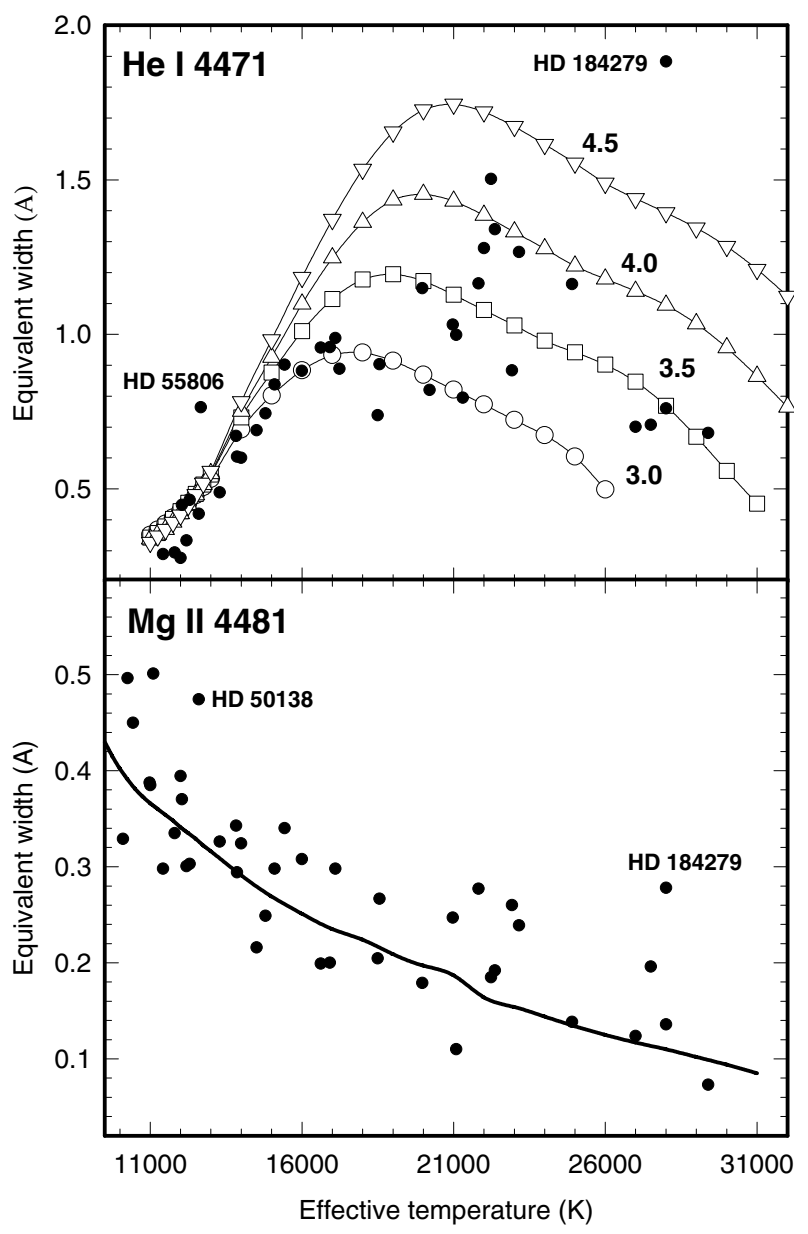

Fig. 1. Computed (curves) and observed (filled circles) equivalent widths for the $\mathrm{He}$ I 4471 (upper panel) and Mg II 4481 (lower panel) spectral lines are shown. The surface gravity adopted in the calculations is noted on each curve. Mg II 4481 has a very weak luminositydependence, for clarity we therefore plotted only the theoretical line corresponding to $\log g=4.0$. Computations are made using planeparallel model atmospheres (Sect. 3).

taken from the ROTINS computer code provided with SYNSPEC (Hubeny \& Lanz 1995). During the fitting procedure, 5 free parameters are considered: the effective temperature $T_{\text {eff }}$, the surface gravity $\log g$, the projected rotation velocity $V \sin i$, the radial velocity $V_{\text {rad }}$ and the wavelength-independent ratio between the mean "flux" level of the normalized observed and theoretical spectra, i.e. a scaling factor allowing to match the stellar continuum. The $\chi^{2}$ parameter is computed in different spectral zones chosen from 4000 to $4500 \AA$. These zones are selected in order to exclude any part of the spectrum that could be affected by line emission or shell absorption (e.g. hydrogen line cores) or/and by interstellar absorption bands (generally found between 4400 and $4450 \AA$ ). As the parameters derived in this way do not take into account the effects of stellar flattening and gravitational darkening due to fast rotation, they will be further called apparent fundamental parameters and correspond to what is obtained when assuming that the star is a sphere with uniform temperature and density surface distributions.

\subsection{Veiling caused by the envelope}

The spectra of Be stars are not only affected by line-emission, but they are also proportionally affected by continuum emission 


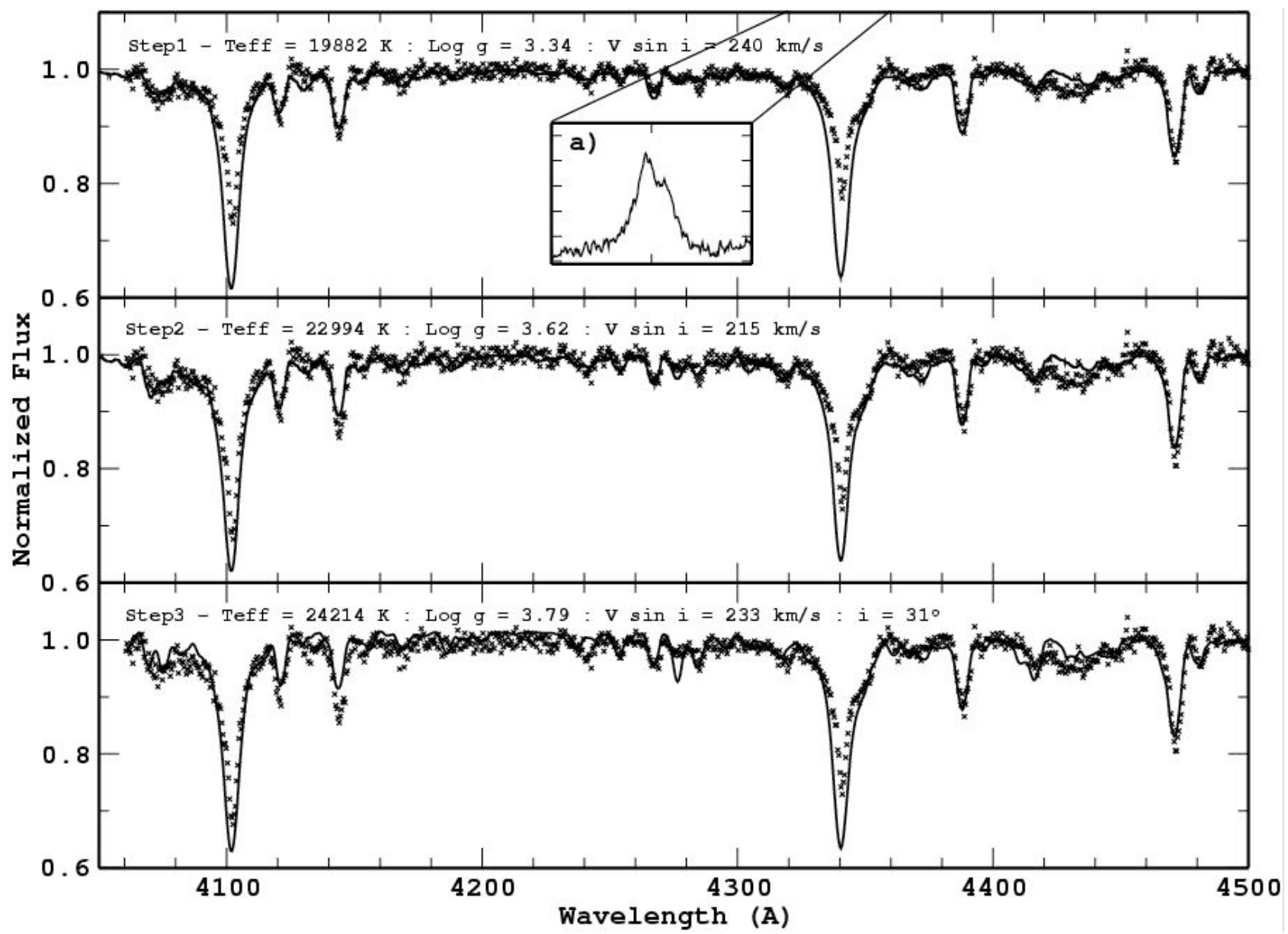

Fig. 2. Description of the three-steps procedure adopted to derive the fundamental parameters. Step 1: fit of the observed spectrum (crosses) with synthetic spectra (solid line), ignoring continuum veiling and second order fast-rotation effects. Subtracting the observed $\mathrm{H} \gamma$ line-profile from the synthetic one allows us to estimate the magnitude of the circumstellar emission (panel a). Step 2: observations are corrected for veiling and fitted again. Step 3: the fundamental parameters derived in step 2 are corrected to account for fast rotation effects with a new fit at $\Omega / \Omega_{\mathrm{c}}=0.99$.

and electron scattering, which change the stellar continuum level. When significant, emission or/and scattering cause an artificial weakening of the spectral line intensity generally leading to underestimation of the effective temperature and of the surface gravity in B-type stars. Ballereau et al. (1995) estimated the magnitude of this veiling by studying hydrogen and helium lines and proposed an empirical approach to correct the stellar spectra for its effects. They mainly introduce a correction term, $r$, which they found directly proportional to the intensity or to the equivalent width of the $\mathrm{H} \gamma$ line-emission $W_{\mathrm{e}}(\mathrm{H} \gamma)$ (see Step 1 of Fig. 2).

To measure the magnitude of the $\mathrm{H} \gamma$ emission, the synthetic $\mathrm{H} \gamma$ profile obtained from a first fit of the fundamental parameters was subtracted from the observations (see Fig. 2). The result was wavelength-integrated in order to obtain $W_{\mathrm{e}}(\mathrm{H} \gamma)$, and to interpolate the $r$ value from Fig. 9a in Ballereau et al. (1995). When greater than zero, this correction was directly applied to the observations (see Eq. (1) in Ballereau et al. 1995), which were finally used to re-derive the fundamental parameters (see Sect. 4.1).

\subsection{Gravitational darkening and stellar flattening}

Be stars are fast rotators with angular velocities probably around $90 \%$ of their breakup velocity. It is expected that a fast and solid-body-type rotation flattens the star, causing a gravitational darkening of the stellar surface due to the variation of the temperature and density distribution from the poles to the equator. For Be stars, we therefore have to account for these effects on the stellar spectra and, consequently, on the determination of the fundamental parameters. In the present paper, these effects are introduced as corrections (see Step 3 of Fig. 2) directly applied to the fundamental parameters derived in the two previous steps (Sects. 4.1 and 4.2). These corrections were estimated by assuming different rotation rates and by systematically comparing a grid of spectra taking into account the effects of fast rotation (Frémat et al. 2004; Frémat et al. 2005) to a grid of spectra computed using the usual plane-parallel model atmospheres (see Sect. 3). Eventually, the complete procedure provides us with the parameters of the stellar parent non-rotating counterpart (i.e. parameters that the stars would have if they were rotationless), further called pnrc parameters, estimated at different $\Omega / \Omega_{\mathrm{c}}$ values. It is the value of these pnrc parameters that should be preferred to interpret and discuss the future COROT data.

\section{Results}

The procedure described in Sect. 3 was applied to the sample of selected Be stars (Table 1). We show in Fig. 3 an example of observed and fitted synthetic spectra limited to a small part of the considered spectral domain. A more complete comparison 

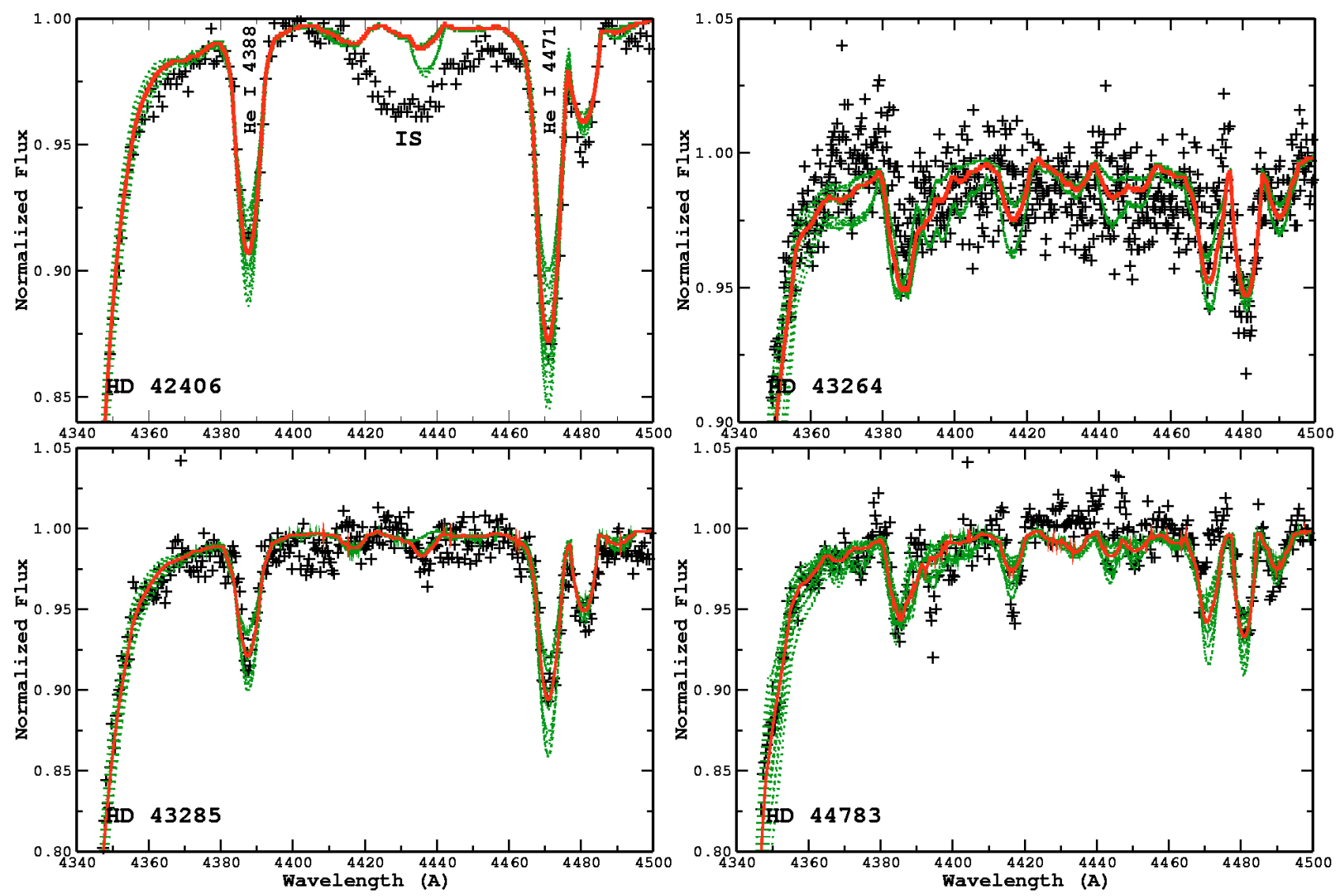

Fig. 3. Comparison between observed data (crosses) and fitted synthetic spectra (full line) in a spectral domain containing the red wing of the $\mathrm{H} \gamma$ line and 2 neutral helium lines. Dotted lines are used to represent 26 spectra computed for different combinations (i.e. there are in fact $3^{3}$ spectra or stellar parameters combinations, but one of them corresponds to the best fit we obtained) of the upper and lower limits of the $T_{\text {eff }}$, $\log g$, and $V \sin i$ values adopting the error bars given in Table 4 .

Table 1. Sample of spectra used for this study. For the ELODIE spectra, the signal to noise ratio $(\mathrm{S} / \mathrm{N})$ was provided by the INTERTACOS $(\mathrm{OHP})$ reduction pipeline, while for the other data it was computed with IRAF by selecting some parts of the continuum in the studied spectral region. " " indicates that the spectrum is available in GAUDI.

\begin{tabular}{llllr}
\hline \hline HD & Obs. date & $T_{\exp }(\mathrm{s})$ & Instrument & $\mathrm{S} / \mathrm{N}$ \\
\hline 42406 & $2004-02-05$ & 900 & FEROS & 249 \\
43264 & $2001-11-27$ & 3300 & ELODIE $^{1}$ & 83 \\
43285 & $2001-12-21$ & 1800 & ELODIE $^{1}$ & 123 \\
44783 & $2000-12-18$ & 1500 & ELODIE $^{1}$ & 121 \\
45901 & $2004-01-03$ & 2700 & AURELIE 4280_G3 120 \\
46380 & $2001-12-22$ & 3600 & ELODIE $^{1}$ & 53 \\
46484 & $2003-01-26$ & 3600 & ELODIE $^{1}$ & 107 \\
47054 & $2002-01-28$ & 300 & FEROS $^{1}$ & 145 \\
\hline
\end{tabular}

performed for all the studied stars is available as a postscript file, which can be downloaded from the CDS. In the same figure, we also plotted with dotted lines 26 synthetic spectra computed for different combinations (i.e. $3^{3}$ spectra or combinations, one of them corresponding to the best fit we obtained) of the upper and lower limits of the $T_{\text {eff }}, \log g$, and $V \sin i$ values adopting the error bars given in Table 4.

We plot in Fig. 4 the apparent value of the effective temperature we derived against the spectral type available in the SIMBAD database. This enables us to compare our determinations with older measurements and to detect any inconsistency (i.e. HD 51404 and HD 184479). Such a comparison is useful
Table 2. Atoms and ions treated in our computations assuming NLTE. The number of levels taken into account for each ion is also given.

\begin{tabular}{lll}
\hline \hline Atom & Ion & Levels \\
\hline Hydrogen & H I & 8 levels + 1 superlevel \\
& H II & 1 level \\
Helium & He I & 24 levels \\
& He II & 20 levels \\
& He III & 1 level \\
Carbon & C II & 53 levels all individual levels \\
& C III & 12 levels \\
& C IV & 9 levels + 4 superlevels \\
Nitrogen & C V & 1 level \\
& N I & 13 levels \\
& N II & 35 levels + 14 superlevels \\
& N III & 11 levels \\
Oxygen & N IV & 1 level \\
& O I & 14 levels + 8 superlevels \\
& O II & 36 levels + 12 superlevels \\
& O III & 9 levels \\
& O IV & 1 level \\
Magnesium & Mg II & 21 levels + 4 superlevels \\
& Mg III & 1 level \\
\hline
\end{tabular}

since, as far as the hydrogen and neutral helium lines are considered, the apparent stellar parameters characterize the spectrum fairly well (e.g. see Fig. 9 in Frémat et al. 2005) and 
Table 3. Theoretical equivalent widths of the main spectral lines found in the considered spectral region and for various spectral types. Computations are made using plane-parallel model atmospheres (Sect. 3). Effective temperatures and spectral types are taken from Gray \& Corbally (1994).

\begin{tabular}{|c|c|c|c|c|c|}
\hline \multirow{3}{*}{ Ion } & \multirow{3}{*}{$\lambda(\AA)$} & \multicolumn{4}{|c|}{ "Equivalent width $(\mathrm{m} \AA)$} \\
\hline & & $\mathrm{B} 0 \mathrm{~V}$ & $\mathrm{~B} 2 \mathrm{~V}$ & B5V & $\mathrm{B} 8 \mathrm{~V}$ \\
\hline & & $29000 \mathrm{~K}$ & $19500 \mathrm{~K}$ & $14000 \mathrm{~K}$ & $11550 \mathrm{~K}$ \\
\hline $\mathrm{He} \mathrm{I}$ & 4009 & 334 & 613 & 217 & 48 \\
\hline $\mathrm{He} \mathrm{I}$ & 4026 & 908 & 1541 & 878 & 542 \\
\hline $\mathrm{HI}_{\mathrm{I}}$ & 4102 & 3869 & 6452 & 9358 & 11475 \\
\hline He I & 4121 & 450 & 251 & 82 & 32 \\
\hline $\mathrm{He} \mathrm{I}$ & 4144 & 443 & 765 & 322 & 120 \\
\hline He I & 4169 & 88 & 91 & 32 & 5 \\
\hline C II & 4267 & 215 & 270 & 97 & 49 \\
\hline $\mathrm{HI}$ & 4340 & 3724 & 6368 & 9552 & 12032 \\
\hline He I & 4388 & 537 & 950 & 378 & 220 \\
\hline $\mathrm{He} \mathrm{I}$ & 4471 & 1087 & 1442 & 667 & 345 \\
\hline Mg II & 4481 & 122 & 198 & 272 & 318 \\
\hline
\end{tabular}

can therefore be directly related to the spectral type given in SIMBAD. Taking into account the fact that our sample also includes stars that have luminosity classes generally ranging from III to $\mathrm{V}$, the distribution of points fairly follows the effective temperature calibration proposed by Gray \& Corbally (1994) for dwarf stars (dashed line in Fig. 4). Table 4 gathers the derived apparent fundamental parameters: Cols. 1 and 2 identify the target; Cols. 3 and 4 respectively give the $V$ magnitude and spectral type extracted from the SIMBAD database; Cols. 5-8 list the effective temperature $\left(T_{\text {eff }}\right)$, surface gravity $(\log g)$, projected rotation velocity $(V \sin i)$ and spectral type we obtained; values found in Cols. 9 and 10 are estimates of the $\mathrm{H} \gamma$ line-emission and continuum veiling due to the presence of the circumstellar envelope; Col. 10 summarizes previous fundamental parameter determinations. The spectral type we give in Col. 8 is an estimate based on the fundamental parameters combined to the $T_{\text {eff }}$ and $\log g$ calibrations proposed by Gray \& Corbally (1994) and by Zorec (1986). Note that, at this stage, our results do not account for stellar flattening and gravitational darkening, but only for veiling. The derived stellar parameters may therefore be considered as veiling-corrected apparent values. We computed the luminosity of each target combining these apparent $T_{\text {eff }}$ and $\log g$ determinations to the theoretical evolutionary tracks of Schaller et al. (1992, $Z=0.02$ ). Their position in the HR diagram is plotted in Fig. 8a. Luminosity accuracy is estimated from the $T_{\text {eff }}$ and $\log g$ error-boxes.

The magnitude of the uncertainty introduced by the fastrotation effects was estimated by applying the approach detailed in Sect. 4.3 (see also Frémat et al. 2005) and by assuming different $\Omega / \Omega_{\mathrm{c}}$ ratios (where $\Omega$ and $\Omega_{\mathrm{c}}$ respectively are the actual and break-up angular velocities). Rotation-corrected fundamental parameters $\left(T_{\mathrm{eff}}^{\mathrm{o}}, \log g_{\mathrm{o}}\right.$ and $\left.V \sin i_{\text {true }}\right)$, i.e. pnrc parameters, and corresponding inclination angles, $i$, are given in Table 5 for $\Omega / \Omega_{\mathrm{c}}=0.8,0.9,0.95$ and 0.99 .

\section{Remarks about specific targets}

\section{1. $H D 43264$}

The Be nature of HD 43264 was first noted by Neiner et al. (2005). However, the surface gravity we derive from the study of the hydrogen and helium lines is quite low (log $g=2.76)$,

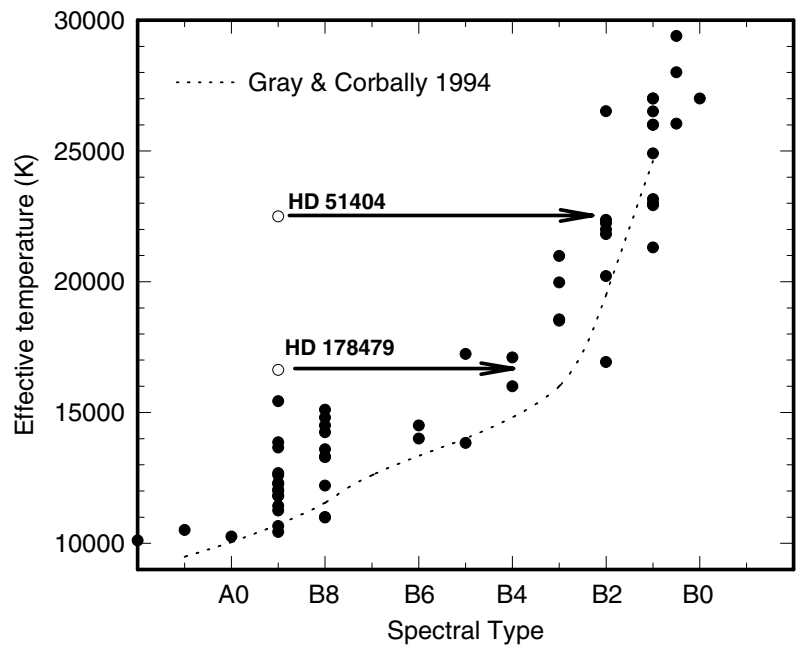

Fig. 4. Veiling-corrected apparent effective temperatures listed in Table 4 versus their spectral type given in SIMBAD (filled circles) and compared to the calibration obtained by Gray \& Corbally (1994) for dwarf stars (dashed line).

which means that the target could be a bright giant. The star is known as a binary in the HIPPARCOS catalogue, probably SB1 considering the magnitude difference between the primary and secondary components $(\Delta V=V \mathrm{~A}-V \mathrm{~B}=-2.85)$.

\section{2. $H D 46380, H D 50087$}

The observed spectra we used to derive the fundamental parameters of HD 46380 and HD 50087 are very noisy, and thus the parameters are uncertain.

\section{3. $H D 50138$}

HD 50138 has a variable shell and is also considered as a $\mathrm{B}[\mathrm{e}]$ star, whose evolutionary status is very difficult to establish. Several hints (Lamers et al. 1998) suggest that the star could be a massive Herbig Be star with an accreting circumstellar disk, i.e. in a pre-main sequence. The spectrum is very complex with strong emission lines and thin absorption features superimposed on the photospheric lines. The determination of fundamental parameters from the fit of the spectrum is therefore very difficult and could be inaccurate.

\section{4. $H D 51404$}

HD 51404 is a poorly studied object recognized as a Be star by Merrill \& Burwell (1949) and erroneously classified as a B9 V star. Our determinations clearly show that its spectral type is B1.5, as can be deduced from Fig. 4 and from the strength of the He I spectral lines.

\subsection{HD 52721}

HD 52721 is also known as a Herbig Ae/Be candidate (Vieira et al. 2003) and is a member of a visual double system with angular separation $\sim 0.65$ arcsec (Perryman et al. 1997). Though a fair agreement between observed and theoretical fitted spectra is observed, the $V \sin i$ value we derive $\left(352 \mathrm{~km} \mathrm{~s}^{-1}\right)$ strongly deviates from those obtained in previous works: $243 \pm 93 \mathrm{~km} \mathrm{~s}^{-1}$ (Yudin 2001) and $456 \mathrm{~km} \mathrm{~s}^{-1}$ (Halbedel 1996). 
Table 4. Veiling corrected apparent stellar parameters. ID numbers, SIMBAD $V$ magnitudes and spectral types are given for each target in Cols. 14. The derived stellar parameters (effective temperature, surface gravity, and $V \sin i$ ) are gathered in Cols. 5-7. Their accuracy is estimated by scanning the solution space while adopting different initial values for the parameters. Spectral types (Col. 8) are derived from the apparent stellar parameters combined to the $T_{\text {eff }}$ and $\log g$ calibrations proposed by Gray \& Corbally (1994) and by Zorec (1986). Columns 9 and 10 list the equivalent width of the $\mathrm{H} \gamma$ emission components and the estimates of the veiling correction, respectively. The error bars on the equivalent widths are generally of the order of $15 \%$ and are a product of the fitting process. Errors on the veiling parameter, $r$, are estimated by accounting for the accuracy on $W_{\lambda}^{\mathrm{H} \gamma}$ and by assuming a 95\% confidence interval on the reference data of Ballereau et al. (Fig. 9a, 1995).

\begin{tabular}{|c|c|c|c|c|c|c|c|c|c|}
\hline \multicolumn{2}{|c|}{$\overline{\mathrm{ID}}$} & \multicolumn{2}{|c|}{ SIMBAD } & \multicolumn{4}{|c|}{ This work } & \multirow[b]{2}{*}{$\begin{array}{c}W_{\lambda}^{\mathrm{H} \gamma} \\
(\mathrm{m} \AA)\end{array}$} & \multirow[b]{2}{*}{$r$} \\
\hline $\mathrm{HD} / \mathrm{BD}$ & $\begin{array}{r}\text { HIP/BD/ } \\
\text { MWC }\end{array}$ & $V$ & Sp. Type & $\begin{array}{l}T_{\text {eff }} \\
(\mathrm{K})\end{array}$ & $\begin{array}{l}\log g \\
\text { (c.g.s.) }\end{array}$ & $\begin{array}{r}V \sin i \\
\left(\mathrm{~km} \mathrm{~s}^{-1}\right)\end{array}$ & Sp. Type & & \\
\hline 42406 & 29298 & 8.01 & B9 & $15400 \pm 1000$ & $3.72 \pm 0.10$ & $300 \pm 25$ & B4 IV & $0.30 \pm 0.05$ & - \\
\hline 43264 & 29719 & 7.51 & B9 & $10500 \pm 1000$ & $2.76 \pm 0.10$ & $288 \pm 10$ & B9 III & $0.22 \pm 0.03$ & - \\
\hline 43285 & 29728 & 6.05 & B6Ve & $14000 \pm 1000$ & $3.78 \pm 0.10$ & $260 \pm 20$ & B5 IV & $0.21 \pm 0.03$ & - \\
\hline 44783 & 30448 & 6.225 & $\mathrm{~B} 8 \mathrm{Vn}$ & $11000 \pm 1000$ & $3.05 \pm 0.15$ & $226 \pm 50$ & B9 III & $0.10 \pm 0.02$ & - \\
\hline 45901 & 30992 & 8.87 & $\mathrm{~B} 2 \mathrm{Ve}$ & $26500 \pm 2000$ & $3.73 \pm 0.15$ & $164 \pm 15$ & B0.5 IV & $0.82 \pm 0.12$ & $0.17 \pm 0.08$ \\
\hline
\end{tabular}

Table 5. Sample of fundamental parameters corrected for the effects of fast rotation at different $\Omega / \Omega_{\mathrm{c}}$ ratios. Error bars on the parameters are of the same order as in Table 4. When the projected rotation velocity is greater than the break-up speed or equatorial speed, the Table is left blank.

\begin{tabular}{|c|c|c|c|c|c|c|c|c|c|c|c|c|c|c|c|c|}
\hline \multirow[b]{2}{*}{ HD } & \multicolumn{4}{|c|}{$\bar{\Omega} \Omega / \Omega_{\mathrm{c}}=0.80$} & \multicolumn{4}{|c|}{$\bar{\Omega} \Omega / \Omega_{\mathrm{c}}=0.90$} & \multicolumn{4}{|c|}{$\overline{\Omega / \Omega_{\mathrm{c}}=0.95}$} & \multicolumn{4}{|c|}{$\bar{\Omega} \Omega / \Omega_{\mathrm{c}}=0.99$} \\
\hline & $\begin{array}{l}T_{\text {eff }}^{\mathrm{o}} \\
(\mathrm{K})\end{array}$ & $\begin{array}{l}\log g_{\mathrm{o}} \\
\text { (cgs) }\end{array}$ & $\begin{array}{l}V \sin i_{\text {true }} \\
\left(\mathrm{km} \mathrm{s}^{-1}\right)\end{array}$ & $\begin{array}{l}i \\
\left({ }^{\circ}\right)\end{array}$ & $\begin{array}{l}T_{\text {eff }}^{\mathrm{o}} \\
(\mathrm{K})\end{array}$ & $\begin{array}{l}\log g_{\mathrm{o}} \\
(\mathrm{cgs})\end{array}$ & $\begin{array}{l}V \sin i_{\text {true }} \\
\left(\mathrm{km} \mathrm{s}^{-1}\right)\end{array}$ & $\begin{array}{l}i \\
\left({ }^{\circ}\right)\end{array}$ & $\begin{array}{l}T_{\text {eff }}^{\mathrm{o}} \\
(\mathrm{K})\end{array}$ & $\begin{array}{l}\log g_{\mathrm{o}} \\
(\mathrm{cgs})\end{array}$ & $\begin{array}{l}V \sin i_{\text {true }} \\
\left(\mathrm{km} \mathrm{s}^{-1}\right)\end{array}$ & $\left({ }^{\circ}\right)$ & $\begin{array}{l}T_{\text {eff }}^{\mathrm{o}} \\
(\mathrm{K})\end{array}$ & $\begin{array}{l}\log g_{\mathrm{o}} \\
\text { (cgs) }\end{array}$ & $\begin{array}{l}\sin i_{\text {true }} \\
\left(\mathrm{km} \mathrm{s}^{-1}\right)\end{array}$ & $\begin{array}{l}i \\
\left({ }^{\circ}\right)\end{array}$ \\
\hline 42406 & & & & & & & & & 16500 & 3.96 & 338 & 61 & 17000 & 3.97 & 360 & 79 \\
\hline 43264 & & & & & & & & & & & & & 12000 & 3.16 & 284 & 79 \\
\hline 43285 & 15000 & 4.03 & 266 & 71 & 15000 & 3.99 & 274 & 55 & 15000 & 3.95 & 292 & 51 & 15000 & 3.93 & 309 & 58 \\
\hline 44783 & 12000 & 3.37 & 226 & 93 & 12000 & 3.40 & 227 & 80 & 11500 & 3.13 & 231 & 82 & 11500 & 3.21 & 218 & 57 \\
\hline 45901 & 27000 & 3.78 & 171 & 31 & 27000 & 3.79 & 173 & 26 & 27500 & 3.84 & 175 & 24 & 29500 & 4.06 & 174 & 21 \\
\hline
\end{tabular}

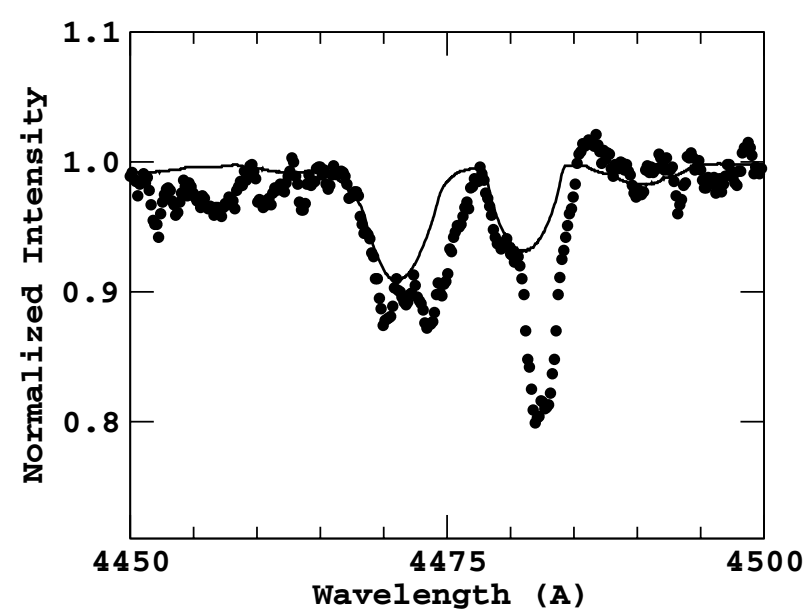

Fig. 5. Observed (dots) and fitted (solid line) He I 4471 and Mg II 4481 lines in the spectrum of HD 55806.

\section{6. $H D 55806$}

HD 55806 is a poorly studied B type star that was found to have bright emission lines by Merrill \& Burwell (1949). We were not able to find any set of fundamental parameters allowing a simultaneous fit of the observed helium and magnesium spectral lines (see Fig. 5). All the helium lines in the studied spectral range show unusual line shapes probably related to the presence of a close companion.

\section{7. $H D 178479$}

Only two publications are found in SIMBAD for HD 178479, which is classified B9 V. The strength of the He I spectral lines is however much too large for a late B-type star and rather corresponds to a B3 star (see Fig. 4).

\subsection{HD 179343}

Thin features superimposed on broader spectral lines are detected in the spectra of HD 179343, which is considered as a Be shell star. It is also known as a "single-entry" binary in the HIPPARCOS catalogue with $\Delta V=0.481$. The shell features could therefore be an artifact of the secondary component.

\section{9. $H D 184279$}

HD 184279 is an early B-type star showing numerous variable shell absorptions superimposed on the photospheric spectrum (Ballereau \& Chauville 1989), as can be noticed from Fig. 1 where the equivalent widths of the He I 4471 and Mg II 4481 lines are clearly overestimated. Since these features are also affecting the helium lines (i.e. our main temperature and $V \sin i$ criteria), the fitting zones were adapted as well as possible to exclude the features.

\section{Discussion}

\subsection{Effects of veiling correction}

Be stars showing strong $\mathrm{H} \gamma$ emission were corrected using an empirical approach described by Ballereau et al. (1995) (see Sect. 4.2). Including such veiling corrections in the calculations 


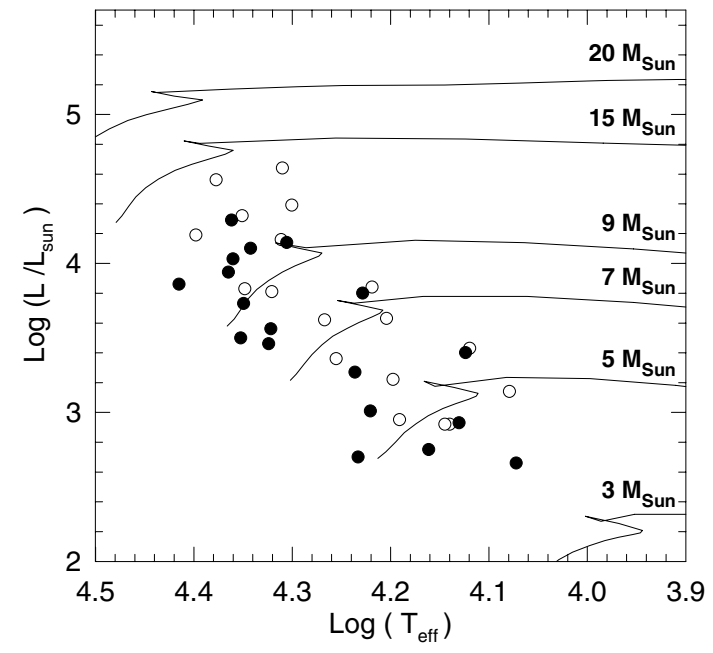

Fig. 6. Location of Be stars showing the strongest $\mathrm{H} \gamma$ emission in the HR diagram with (filled circles) and without (open circles) accounting for the veiling effects. Theoretical evolutionary tracks are taken from Schaller et al. (1992) for a solar-like metallicity.

Table 6. Average $V \sin i$ in $\mathrm{km} \mathrm{s}^{-1}$ computed assuming different $\Omega / \Omega_{\mathrm{c}}$ towards the Galactic centre and anticentre. For each value of $\Omega / \Omega_{\mathrm{c}}$, only a certain number of stars (given in brackets) have a reasonable model (see Table 5) and could be used in the computation.

\begin{tabular}{ccc}
\hline \hline & Anticentre & Centre \\
$\Omega / \Omega_{\mathrm{c}}$ & B9-B0 & B9-B0 \\
\hline 0.80 & $221 \pm 65(27)$ & $188 \pm 75(27)$ \\
0.90 & $236 \pm 75(29)$ & $216 \pm 79(30)$ \\
0.95 & $251 \pm 84(31)$ & $223 \pm 82(30)$ \\
0.99 & $260 \pm 92(32)$ & $235 \pm 84(31)$ \\
\hline
\end{tabular}

often produces a lowering of the observed continuum and, consequently, a spectral line strengthening. From this procedure, different $T_{\text {eff }}$ and higher $\log g$ values are generally obtained, which in the HR diagram pulls the location of the Be stars towards the ZAMS (see Fig. 6).

\subsection{Effects of fast rotation}

In our sample, the effects of fast rotation on effective temperature, surface gravity and projected rotation velocity are generally significant when $V \sin i>150 \mathrm{~km} \mathrm{~s}^{-1}$. As expected, accounting for these effects provides higher values of the pnrc fundamental parameters (e.g. average $V \sin i$ in Table 6). We further note from Table 5 that, for $\Omega / \Omega_{\mathrm{c}} \geq 0.8$ and if we except $V \sin i_{\text {true }}$, the pnrc stellar parameters generally do not vary significantly with $\Omega / \Omega_{\mathrm{c}}$. This is mainly due to the fact that, at a fixed $V \sin i$ value, the increase of $\Omega / \Omega_{\mathrm{c}}$ leads to smaller inclinations and, consequently, leads to exploring regions of the stellar surface that are less affected by the flattening of the star. Since a recent study (Frémat et al. 2005) showed that Be stars are found to rotate, on average, at $\Omega / \Omega_{\mathrm{c}} \sim 0.88$ when fast rotation effects are properly treated, uncertainties on the actual value of the angular velocity are not expected to induce too high errors on the estimate of the pnrc $T_{\text {eff }}^{\mathrm{o}}$ and $\log g_{\mathrm{o}}$ parameters.

From Fig. 7, we see that the effects of fast rotation affect the target spectral type and luminosity class. As already mentioned by Briot \& Zorec (1994), the top of the spectral type distribution of Be stars is centered on B1 (instead of B2) when gravitational darkening and stellar flattening are taken into account. The luminosity class of Be stars is even more sensitive to these effects, which shift the targets toward lower luminosities when they are included in the computations. In view of these results, and keeping in mind that the veiling effect makes this situation even worse, it seems that luminosity classes are very inaccurate in characterizing the evolutionary status of Be stars.

\subsection{Evolutionary status of Be stars}

The knowledge of whether the Be phenomenon is an innate property of the stars or whether it depends on stellar evolution is a matter of great interest to better understand the precise nature of Be stars and its potential link with asteroseismology. A recent study (Zorec et al. 2005) performed on a large sample of field Be stars (i.e. 97 Be stars) uniformly spread over the whole sequence of B type stars showed that the appearance of the Be phenomenon for the lower mass stars generally occurs during the second half of the main sequence life time $\left(\tau_{\mathrm{MS}}\right)$, but that it appears earlier at greater stellar masses. In order to see if there is such a trend in our sample of Be stars, we derive their masses, ages and luminosity using an interpolation procedure developed by Zorec et al. (2005), which accounts for the changes introduced by fast rotation in the evolutionary tracks (Heger \& Langer 2000; Meynet \& Maeder 2000, 2002; Maeder \& Meynet 2001). Since these modeled evolutionary tracks are given in terms of surface-averaged effective temperatures and bolometric luminosities, we transformed the pnrc parameters into surfaceaveraged ones using the angular velocity ratios $\Omega / \Omega_{\mathrm{c}}=0.80$, 0.90 , and 0.99 . The pnrc surface-averaged parameters and the respective interpolated masses $M / M_{\odot}$, ages $\tau$, and fractional ages $\tau / \tau_{\text {MS }}$ are given in Table 7 . Figure 8 a shows the HR diagram of the program stars depicted by the veiling-corrected apparent $T_{\text {eff }}$ and $\log L / L_{\odot}$ values and evolutionary tracks for non-rotating stars (Schaller et al. 1992). Figures 8b-8d show the HR diagram of the program stars in terms of surface-averaged fundamental parameters assuming the stars rotate at $\Omega / \Omega_{\mathrm{c}}=$ $0.80,0.90$ and 0.99 respectively. The corresponding evolutionary tracks (Figs. 8b-8d) were calculated for the ZAMS equatorial rotation velocity $V_{\mathrm{o}}=300 \mathrm{~km} \mathrm{~s}^{-1}$. Note that $\Omega / \Omega_{\mathrm{c}}=0.90$ represents the average angular velocity rate of galactic field Be stars (Frémat et al. 2005). If a star starts its evolution on the ZAMS as a rigid rotator with a rotation velocity $V_{\mathrm{o}}$, as a consequence of the initial angular momentum redistribution, the surface velocity decreases somewhat in a time ranging from 1 to $2 \%$ (Denissenkov et al. 1999) of the stellar $\tau_{\text {MS }}$. Since, on one hand, we do not know the actual initial velocity of the sample stars on the ZAMS and, on the other hand, dwarf Be stars have a very flat distribution of their equatorial true rotational velocities versus the spectral type around $V \sim 300 \mathrm{~km} \mathrm{~s}^{-1}$ (Yudin 2001; Zorec et al. 2004, Fig. 1b), the comparisons done above with evolutionary tracks calculated for $V_{\mathrm{o}}=300 \mathrm{~km} \mathrm{~s}^{-1}$ are realistic (Meynet \& Maeder 2000).

Figure 9 shows the average $\tau / \tau_{\mathrm{MS}}$ ratios we computed for each star assuming $\Omega / \Omega_{\mathrm{c}}=0.90$. Most of the Be stars are found, on average, in the second half of the main sequence life time and we clearly observe a paucity of very young Be stars at masses lower than $6 M_{\odot}$. The lack of low mass Be stars $\left(M<7 M_{\odot}\right)$ in the first half of the main sequence was noted in other recent works that deal with independent stellar samples. Using a magnitude-limited sample (97 Be stars) that mirrors the distribution of all known Be stars in the Bright Stars Catalogue (Hoffleit \& Warren 1991), Zorec et al. (2005) obtained a void 

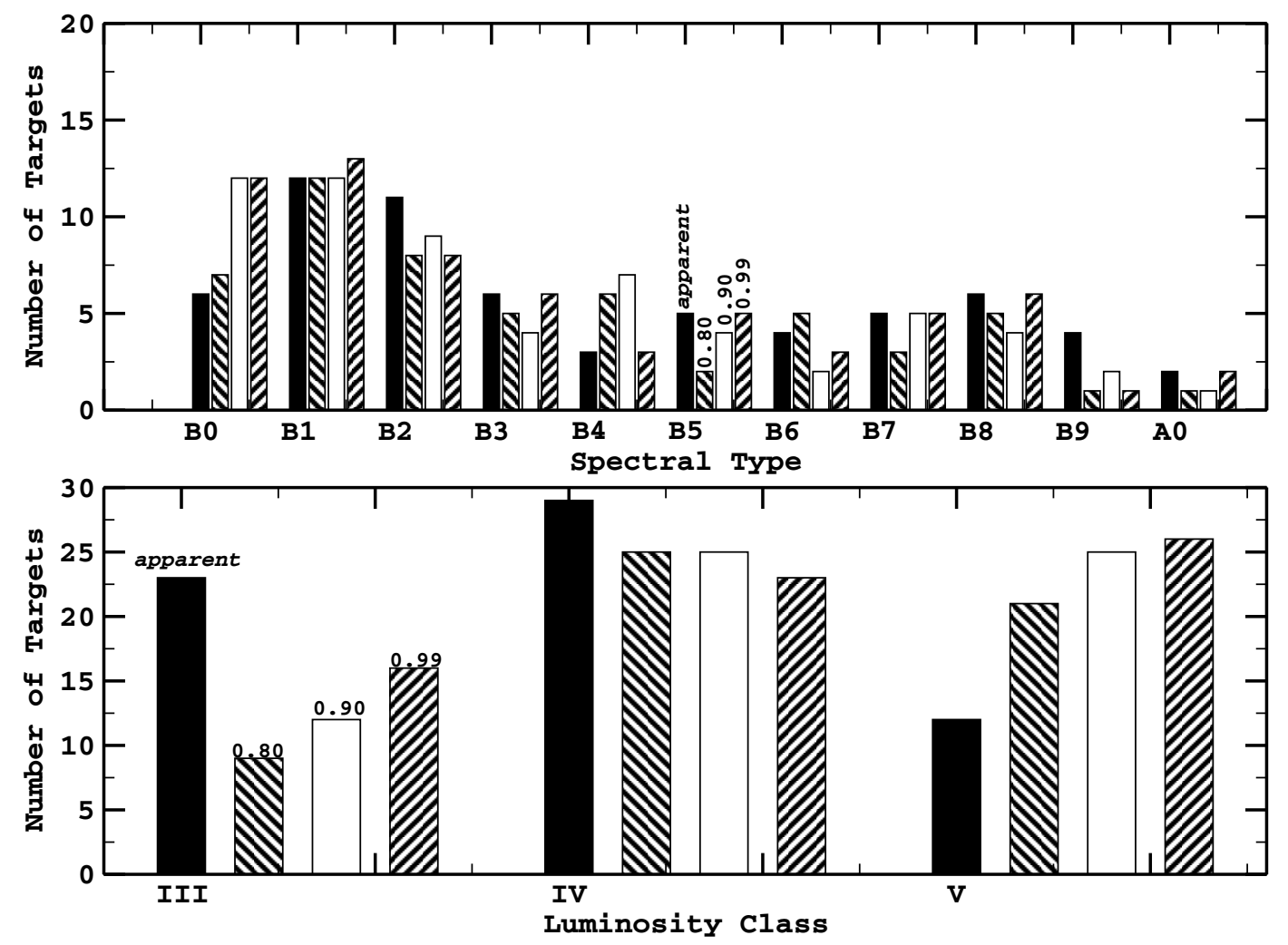

Fig. 7. Histogram showing the changes in spectral type and luminosity class distribution when including the effects of stellar flattening and gravitational darkening. Apparent spectral types and luminosity classes are represented with filled bars, while other bars give the corrected ones adopting $\Omega / \Omega_{\mathrm{c}}=0.80,0.90,0.99$.

Table 7. Sample of the pnrc surface-averaged parameters and of the respective interpolated masses $M / M_{\odot}$, ages $\tau$, and fractional ages $\tau / \tau_{\mathrm{MS}}$. When the projected rotation velocity was greater than the break-up speed or than the equatorial speed, the Table were left blank.

\begin{tabular}{|c|c|c|c|c|c|c|}
\hline \multicolumn{7}{|c|}{$\Omega / \Omega_{\mathrm{c}}=0.80$} \\
\hline \multicolumn{7}{|l|}{42406} \\
\hline \multicolumn{7}{|l|}{43264} \\
\hline 43285 & $14500 \pm 1100$ & $4.02 \pm 0.17$ & $2.69 \pm 0.09$ & $4.60 \pm 0.30$ & $6.6 \mathrm{E}+07 \pm 2.0 \mathrm{E}+07$ & $0.47 \pm 0.13$ \\
\hline 44783 & $11500 \pm 1100$ & $3.33 \pm 0.21$ & $2.94 \pm 0.12$ & $4.30 \pm 0.30$ & $1.7 \mathrm{E}+08 \pm 1.5 \mathrm{E}+07$ & $1.02 \pm 0.03$ \\
\hline 45901 & $26000 \pm 2300$ & $3.74 \pm 0.21$ & $4.43 \pm 0.13$ & $13.60 \pm 1.30$ & $1.2 \mathrm{E}+07 \pm 2.0 \mathrm{E}+06$ & $0.75 \pm 0.10$ \\
\hline 46380 & $23000 \pm 1100$ & $3.88 \pm 0.14$ & $3.94 \pm 0.12$ & $10.00 \pm 0.60$ & $1.7 \mathrm{E}+07 \pm 2.6 \mathrm{E}+06$ & $0.62 \pm 0.09$ \\
\hline 46484 & $27000 \pm 1000$ & $3.64 \pm 0.13$ & $4.65 \pm 0.14$ & $15.70 \pm 1.30$ & $1.1 \mathrm{E}+07 \pm 6.0 \mathrm{E}+05$ & $0.80 \pm 0.04$ \\
\hline 47054 & $12500 \pm 800$ & $3.58 \pm 0.15$ & $2.87 \pm 0.12$ & $4.60 \pm 0.20$ & $1.3 \mathrm{E}+08 \pm 1.1 \mathrm{E}+07$ & $0.91 \pm 0.05$ \\
\hline 47160 & $11500 \pm 600$ & $3.74 \pm 0.11$ & $2.41 \pm 0.08$ & $3.60 \pm 0.10$ & $2.1 \mathrm{E}+08 \pm 1.7 \mathrm{E}+07$ & $0.79 \pm 0.05$ \\
\hline \multicolumn{7}{|l|}{47359} \\
\hline 49330 & $26000 \pm 1700$ & $3.84 \pm 0.17$ & $4.30 \pm 0.12$ & $12.80 \pm 1.00$ & $1.2 \mathrm{E}+07 \pm 1.9 \mathrm{E}+06$ & $0.69 \pm 0.10$ \\
\hline
\end{tabular}

of low mass Be stars in the first half of the main sequence evolutionary phase. An equivalent conclusion was also obtained by Levenhagen (2004) from a study of 130 Be stars with visual magnitudes ranging from 7 to 9 mag. In our case, probably due to the fact that the sample is smaller and the stars are not uniformly distributed over the mass range that defines B-type stars, the trend is mainly obvious at masses $<6 M_{\odot}$. However, since the same result also repeats in other studies, we think that this effect is unlikely to be related to some sampling bias.

Furthermore, if we exclude the targets with low $\mathrm{S} / \mathrm{N}$ spectra, 5 stars (HD 50868, HD 51404, HD 52721, HD 174571, BD $\left.-9^{\circ} 4858\right)$ are obviously much younger than the rest of the sample (Fig. 9) and might be considered as Herbig Ae/Be candidates, as is already the case for HD 174571 (Vieira et al. 2003).

\subsection{Be stars as COROT targets}

Fields to be observed with COROT have already been preselected based on the parameters of the potential targets. Only Be stars located in these fields can still be chosen as secondary targets and would then be observed for about 5 consecutive months. Table 8 summarizes these Be stars that are candidates as secondary targets. Whether all these fields will be observed with COROT will depend on the length of the mission; at least five of them will be observed. Moreover, since COROT tries to sample as well as possible the HR diagram, not all the secondary Be candidates in a selected field will eventually be observed with COROT. The choice will be made according to technical constraints of the satellite and to the stellar parameters determined in this paper. 


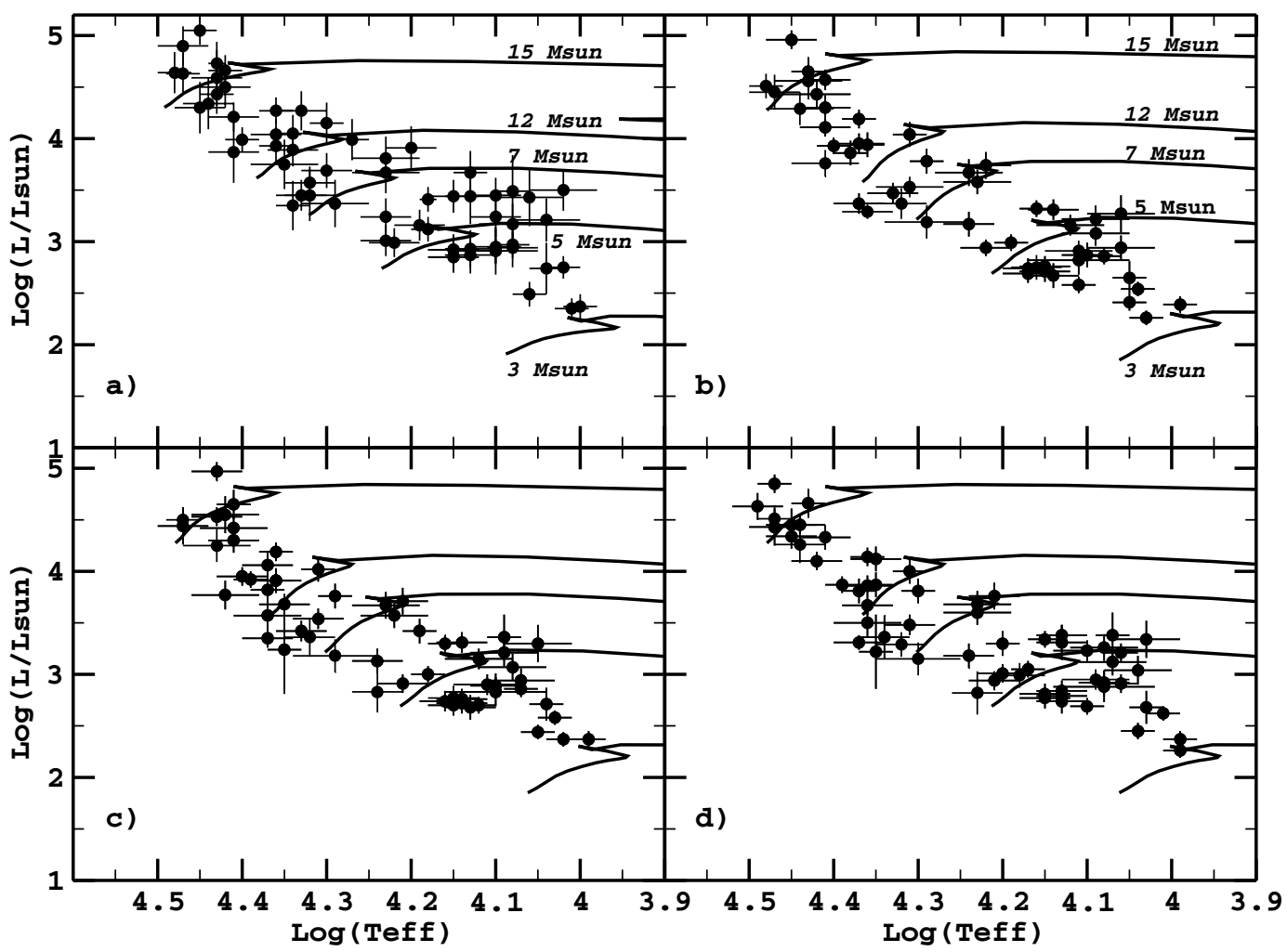

Fig. 8. Panel a): location of the Be stars (filled circles) in the HR diagram adopting the veiling-corrected apparent stellar parameters of Table 4. Theoretical evolutionary tracks (lines) are taken from Schaller et al. (1992) and adapted following a procedure given by Zorec et al. (2005). Panels b), c) and d): location of the Be stars (filled circles) in the HR diagram accounting for gravitational darkening effects and assuming $\Omega / \Omega_{\mathrm{c}}=$ $0.80,0.90$ and 0.99 , respectively. Theoretical evolution tracks (lines) take into account the effects of fast rotation as described by Meynet $\&$ Maeder (2000). The effective temperature and the luminosity reported in the figures are therefore surface-averaged quantities (Table 7).

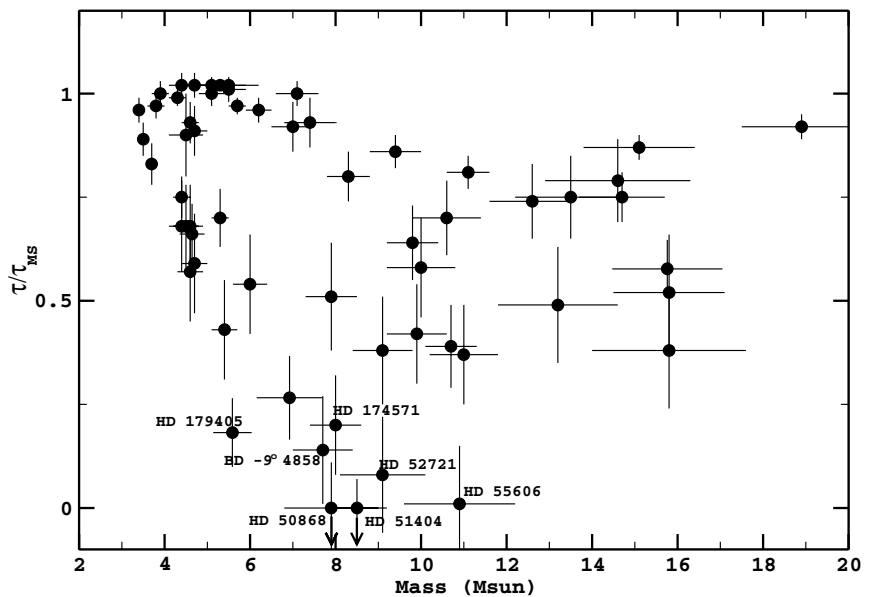

Fig. 9. $\tau / \tau_{\mathrm{MS}}$ ratios of the program Be stars as a function of stellar mass (see Table 7). The ratios and masses are computed for $\Omega / \Omega_{\mathrm{c}}=0.90$, which corresponds to the expected average angular velocity of Be stars.

Be stars that will not be observed in a long run, either because they are not located in one of the selected fields (and therefore are not listed in Table 8) or because they are not chosen as a secondary target in these fields, can be proposed as targets for short runs lasting 20-30 days. Again the choice of these Be short-run targets will depend on the fundamental parameters determined in this paper, and on their variability. In particular HD 168797 seems to be a prime candidate for a short-run (see Gutiérrez-Soto et al. 2005).
Table 8. Be stars close to primary COROT target candidates, which could be selected as secondary targets.

\begin{tabular}{llll}
\hline \hline \multirow{4}{l}{ Galactic Centre } \\
Be Secondary & V & Sp. Type & COROT Field \\
\hline HD 171219 & 7.65 & B5 III & HD 171234 and HD 170580 \\
HD 171219 & 7.65 & B5 III & HD 171834 \\
HD 175869 & 5.56 & B8 III & HD 175726 \\
HD 181231 & 8.58 & B5 IV & HD 181555 and HD 180642 \\
\hline \multicolumn{5}{c}{ Galactic Anticentre } \\
Be Secondary & V & Sp. Type & COROT Field \\
\hline HD 43285 & 6.05 & B5 IV & HD 43587 \\
HD 43913 & 7.88 & A0 & HD 43587 \\
HD 45901 & 8.87 & B0.5 IV & HD 46558 \\
HD 46484 & 7.65 & B0.5 IV & HD 46558 \\
HD 47359 & 8.87 & B0 IV & HD 46558 \\
HD 49330 & 8.95 & B0.5 IV & HD 49933 and HD 49434 \\
HD 49567 & 6.15 & B3 III & HD 49933 and HD 49434 \\
HD 49585 & 9.13 & B0.5 IV & HD 49933 and HD 49434 \\
HD 50087 & 9.08 & B8 III & HD 49933 and HD 49434 \\
HD 50209 & 8.36 & B8 IV & HD 49933 and HD 49434 \\
HD 50891 & 8.88 & B0.5 V & HD 52265 \\
HD 51193 & 8.06 & B1.5 IV & HD 52265 \\
HD 51404 & 9.30 & B1.5 V & HD 52265 \\
HD 51452 & 8.08 & B0 IV & HD 52265 \\
\hline
\end{tabular}

\section{Conclusions}

In this paper, we determined the fundamental parameters (spectral type, effective temperature, surface gravity and projected 
rotation velocity) for the Be stars that can be observed in COROT's seismology fields. To this end, a careful and detailed modeling of the stellar spectra was applied, accounting for NLTE effects as well as for the effects due to fast rotation (stellar flattening and gravitational darkening). On average, the uncertainties we obtained on the stellar parameters are of the order of $10 \%$ on $V \sin i, 7 \%$ on $T_{\text {eff }}$, and $3.3 \%$ on $\log L / L_{\odot}$. Our results are therefore very well suited for the target selection of COROT Be stars and, further, will also be very useful when analyzing the huge amount of data that COROT will provide.

A study of the fast rotation effects, as well as of those related to circumstellar veiling, on the spectral type and luminosity class distribution shows that the luminosity class of Be stars is generally inaccurate in characterizing their evolutionary status. Evolutionary tracks for fast rotators were further used to derive stellar masses and ages in order to discuss the appearance of the Be phenomenon with respect to the evolutionary status of the stars. Most of the Be stars of our sample are found in the second half of the main sequence life time. There is further an obvious lack of very young Be stars at masses lower than $6 M_{\odot}$ which reproduces previous observations made by Zorec et al. (2005) and Levenhagen (2004).

Finally, we give a preliminary list of the Be stars that could be chosen as secondary targets for the COROT mission. The fundamental stellar parameters we derived will be further used to proceed with the secondary target selection procedure, as well as to determine which objects could be proposed for short runs.

Acknowledgements. We wish to acknowledge the referee, D. Baade, for his suggestions which greatly improved the paper. We thank J.Gutiérrez-Soto for his remarks. We are grateful to the ground-based asteroseismology working group of COROT and all the contributors of GAUDI. This research has made use of the SIMBAD database maintained at CDS, Strasbourg, France. YF thanks Dr. P. Lampens for hosting him at the Royal Observatory of Belgium. JRM acknowledges continuous financial support of the CNPQ Brazilian Agency.

\section{References}

Abt, H. A., Levato, H., \& Grosso, M. 2002, ApJ, 573, 359

Baglin, A., Auvergne, M., Catala, C., et al. 2002, in IAU Coll. 185: Radial and Nonradial Pulsations as Probes of Stellar Physics, ASP Conf. Ser., 259, 626

Ballereau, D., \& Chauville, J. 1989, A\&A, 214, 285

Ballereau, D., Chauville, J., \& Zorec, J. 1995, A\&AS, 111, 423

Briot, D., \& Zorec, J. 1994, in Pulsation; Rotation; and Mass Loss in Early-Type Stars, IAU Symp., 162,356
Chauville, J., Zorec, J., Ballereau, D., et al. 2001, A\&A, 378, 861

Cidale, L., Zorec, J., \& Tringaniello, L. 2001, A\&A, 368, 160

Conti, P. S., \& Ebbets, D. 1977, ApJ, 213, 438

Cunto, W., Mendoza, C., Ochsenbein, F., \& Zeippen, C. J. 1993, A\&A, 275, L5

Denissenkov, P. A., Ivanova, N. S., \& Weiss, A. 1999, A\&A, 341, 181

Floquet, M., Neiner, C., Janot-Pacheco, E., et al. 2002, A\&A, 394, 137

Frémat, Y., Zorec, J., Hubert, A. M., et al. 2004, in IAU Symp., 23

Frémat, Y., Zorec, J., Hubert, A.-M., \& Floquet, M. 2005, A\&A, 440, 305

Gray, R. O., \& Corbally, C. J. 1994, AJ, 107, 742

Grevesse, N., \& Sauval, A. J. 1998, Space Sci. Rev., 85, 161

Gulati, R. K., Malagnini, M. L., \& Morossi, C. 1989, A\&AS, 80, 73

Gutiérrez-Soto, J., Fabregat, J., Suso, J., et al. 2005, A\&A, in preparation

Halbedel, E. M. 1996, PASP, 108, 833

Heger, A., \& Langer, N. 2000, ApJ, 544, 1016

Hoffleit, D., \& Warren, J. W. H. 1991, The Bright Star Catalogue, 5th Revised Ed. (Astronomical Data Center, NSSDC/ADC)

Hubeny, I., \& Lanz, T. 1995, ApJ, 439, 875

Hubert, A. M., \& Floquet, M. 1998, A\&A, 335, 565

Jaschek, M., Slettebak, A., \& Jaschek, C. 1981, Be stars Newsletter, 4, 9

Kurucz, R. L. 1993, Kurucz CD-ROM No.13. Cambridge, Mass.: Smithsonian Astrophysical Observatory.

Lamers, H. J. G. L. M., Zickgraf, F., de Winter, D., Houziaux, L., \& Zorec, J. 1998, A\&A, 340,117

Lanz, T., \& Hubeny, I. 2003, ApJS, 146, 417

Levenhagen, R. 2004, Ph.D. Thesis (University of Sao Paulo)

Levenhagen, R. S., \& Leister, N. V. 2004, AJ, 127, 1176

Lyubimkov, L. S., Rachkovskaya, T. M., Rostopchin, S. I., \& Lambert, D. L. 2002, MNRAS, 333,9

Maeder, A., \& Meynet, G. 2001, A\&A, 373, 555

Merrill, P. W., \& Burwell, C. G. 1949, ApJ, 110, 387

Meynet, G., \& Maeder, A. 2000, A\&A, 361, 101

Meynet, G., \& Maeder, A. 2002, A\&A, 390, 561

Neiner, C., Hubert, A.-M., Floquet, M., et al. 2002, A\&A, 388, 899

Neiner, C., Hubert, A.-M., \& Catala, C. 2005, ApJS, 156, 237

Perryman, M. A. C., Lindegren, L., Kovalevsky, J., et al. 1997, A\&A, 323, L49

Porter, J. M., \& Rivinius, T. 2003, PASP, 115, 1153

Rivinius, T., Baade, D., Stefl, S., et al. 1998, A\&A, 336, 177

Royer, F., Grenier, S., Baylac, M.-O., Gómez, A. E., \& Zorec, J. 2002, A\&A, 393, 897

Schaller, G., Schaerer, D., Meynet, G., \& Maeder, A. 1992, A\&AS, 96, 269

Sigut, T. A. A. 1996, ApJ, 473, 452

Slettebak, A. 1982, ApJS, 50, 55

Sokolov, N. A. 1995, A\&AS, 110, 553

Solano, E., Catala, C. Garrido, R., et al. 2005, AJ, 129, 547

Theodossiou, E. 1985, MNRAS, 214, 327

Townsend, R. H. D., Owocki, S. P., \& Howarth, I. D. 2004, MNRAS, 350

Varosi, F., Lanz, T., de Koter, A., et al. 1995,

ftp://idlastro.gsfc.nasa.gov/pub/contrib/varosi/modion

Vieira, S. L. A., Corradi, W. J. B., Alencar, S. H. P., et al. 2003, AJ, 126, 2971

Yudin, R. V. 2001, A\&A, 368, 912

Zorec, J. 1986, Ph.D. Thesis: Structure et rotation différentielle dans les étoiles B avec et sans émission (Paris: Université VII, 1986)

Zorec, J., Levenhagen, R., Chauville, J., et al. 2004, in IAU Symp., 89

Zorec, J., Frémat, Y., \& Cidale, L. 2005, A\&A, 441, 235 